\title{
A survey of Korean medicine doctors' clinical practice patterns for autism spectrum disorder: preliminary research for clinical practice guidelines
}

\author{
Jihong Lee ${ }^{1,2}$, Sun Haeng Lee ${ }^{1,3}$, Boram Lee ${ }^{1,2}$, In Jun Yang ${ }^{4}$ and Gyu Tae Chang ${ }^{1,2^{*}}$ (D)
}

\begin{abstract}
Background: The aim of this study was to investigate autism spectrum disorder (ASD) clinical practice patterns of Korean medicine doctors (KMDs) through questionnaire survey.

Methods: Questionnaires on Korean medicine (KM) treatment for ASD were distributed to $255 \mathrm{KMDs}$ on December 5, 2016. The KMDs were psychiatrists, pediatricians, or general practitioners, who treated patients with ASD. The questionnaire covered items on treatment methods, aims of treatment, KM syndrome differentiation, diagnostic tools, and sociodemographic characteristics. Frequency analysis was conducted to describe the participants and their practices.

Results: A total $22.4 \% \mathrm{KMDs}(n=57 / 255)$ completed the questionnaires and $54 \mathrm{KMDs}(21.2 \%)$ matched the inclusion criteria. The KMDs utilized herbal medicine (27.3\%), body acupuncture (17.6\%), scalp acupuncture (10.7\%), moxibustion (6.4\%), and Korean medical psychotherapy (5.9\%) to treat ASD. The most commonly prescribed herbal medicine was Yukmijihwang-tang. Forty-eight (88.9\%) KMDs responded that they used KM syndrome differentiation. 'Organ system, Qi, Blood, Yin, Yang, Fluid and Humor diagnosis' was most frequently used for syndrome differentiation. ASD was mainly diagnosed based on the fourth edition of the Diagnostic and Statistical Manual for Mental Disorders (DSM-IV) and DSM-5.

Conclusions: The present study demonstrated the current status of KMDs' diagnosis and treatment of ASD. In future clinical trials and clinical practice guidelines, these findings will provide meaningful information on the actual practice patterns of KMDs.
\end{abstract}

Keywords: Autism spectrum disorder, Health surveys, Korean traditional medicine, Complementary therapies

\section{Background}

Autism spectrum disorder (ASD) is a lifelong, neurodevelopmental disorder characterized by deficits in social interaction and communication, and restricted and repetitive patterns of behavior, interests, and activities [1]. Approximately 1 in 68 children has been identified with ASD in the United States, with an average prevalence of

\footnotetext{
* Correspondence: gtchang@khu.ac.kr

'Department of Clinical Korean Medicine, Graduate School, Kyung Hee University, Seoul 02453, Republic of Korea

${ }^{2}$ Department of Pediatrics of Korean Medicine, Kyung Hee University Hospital at Gangdong, \#892 Dongnam-ro, Gangdong-gu, Seoul 05278, Republic of Korea

Full list of author information is available at the end of the article
}

$1-2 \%$ in Asia, Europe, and North America [2]. The prevalence of ASD in a total population sample of South Korean children aged 7-12 years was estimated to be $2.64 \%$ [3]. There is no known widely accepted treatment method, but interventions may improve symptoms. Treatment modalities for ASD are categorized as behavioral and educational interventions, psychopharmacologic interventions, and complementary and alternative medicine (CAM) [4].

Korean medicine (KM) has developed similarly to East Asian traditional medicine, such as traditional Chinese medicine and Oriental medicine in Japan [5]. In parallel with the medical system in South Korea, Korean 
medicine doctors (KMDs) are licensed to independently practice all main curative methods of Korean medical treatments, unlike medical doctors (MDs) who utilize conventional medicine $[6,7]$. The main KM therapeutic methods include acupuncture, moxibustion, cupping, herbal medicines, and manual therapies [6]. The KM training system is similar to that of conventional medicine, with 6 years of undergraduate education and a national licensing examination [8]. In 2013, there were 21,355 KMDs in South Korea. The percentage of KMDs among total doctors (including KMDs, MDs, and dental doctors) is $13.5 \%$ [9]. The KM specialist training system was introduced in 1999 by the Ministry of Health and Welfare, with reference to the conventional medicine specialist system. After getting a KM license, KMDs choose to train in a designated KM hospital for 4 years (1-year internship and 3-year residence course) and can obtain specialist certification [6]. KMDs with specialist license accounted for 14.3\% of all KMDs by 2015 [10]. The types of specialty subjects include internal medicine, acupuncture and moxibustion, rehabilitation medicine, pediatrics, gynecology, ophthalmology, otorhinolaryngology, dermatology, neuropsychiatry, and Sasang constitutional medicine. In 2012, the medical expenses for KM were 4 billion USD, and the proportion of National Health Expenditure was 4.5\% [11].

Clinical practice guidelines (CPGs) assist healthcare providers and patients in making treatment decisions [12]. A CPG for ASD has not yet been established in both KM and conventional medicine fields in Korea. KMDs use herbal medicine and acupuncture to treat patients with ASD [13, 14]. However, KM treatment for ASD is not standardized, since KMDs use different KM syndrome differentiations and therapeutic methods. KMDs have difficulty obtaining conclusive evidence for practice, and thus, there is a need to develop evidencebased CPGs for ASD that follow a systematic methodology. The CPGs are required to reflect the current state of KM treatment. The aim of the current study was to investigate ASD clinical practice patterns of KMDs through a questionnaire survey.

\section{Methods}

\section{Subjects}

We conducted a survey targeting KM pediatrics and neuropsychiatry specialists, and general practitioners who stated that they treat patients with ASD. KM pediatrics professors or KM neuropsychiatry professors at KM universities in Korea were also included. We contacted KM pediatrics and KM neuropsychiatrists through the Society of Korean Medicine Pediatrics and Society of Korean Medicine Neuropsychiatry, respectively. In addition, to avoid missing KMDs who treat ASD among other specialists or general practitioners, we performed an additional internet search. To identify KMDs who treat ASD, we searched Google [15], Naver [16], and Daum [17], which are Korean internet search engines, in September 2016. The search phrases, in Korean, were "Autism AND Korean Medicine" and "Autism AND Korean Medicine Clinic". We visited the websites of the KM clinics to identify pediatric or psychiatric disease clinics, and ascertained whether they provide medical care to patients with ASD. Information about KMDs and KM clinics were collected from the website. Respondents stating that they had no experience in ASD outpatient treatment or for whom we could not obtain the consent form were excluded from the analysis.

\section{Initial draft}

The questionnaire was constructed after a comprehensive literature review and reviewed by KMD researchers. Four participating KMDs had completed 6-year undergraduate courses. One of them (CGT) is a KM pediatrics specialist with more than 20 years of clinical experience and is a professor of KM pediatrics. Two KMDs (LJ and LSH) are KM pediatrics specialists with more than 8 years of clinical experience. The fourth KMD is a resident (LB) majoring in KM pediatrics with more than 2 years of clinical experience. The questionnaire covered items on treatment methods, aims of treatment, KM syndrome differentiation, diagnostic tools, and sociodemographic characteristics. Items regarding KM syndrome differentiation and non-pharmacological treatments were based on the KM neuropsychiatry textbook [18]. One question regarding the division of patient-age group was based on the KM pediatrics textbook [19].

\section{Second draft}

A panel of six extramural experts reviewed and provided advice on the initial draft. The panel was composed of 2 $\mathrm{KM}$ pediatric professors at KM universities who were licensed KMDs and four KMDs who mainly treated children or adults with neuropsychiatric disease at KM clinics. One had a Master's in public health. Four researchers revised the questionnaire based on comments from the panel. The survey questions were finalized after two conferences addressing revisions. An additional file shows the full survey [see Additional file 1].

\section{Distribution and collection of questionnaires}

The questionnaires were distributed and collected directly, by e-mail or registered mail, at the conference site of the Society of Korean Medicine Pediatrics. The emails to KM pediatrics and neuropsychiatry specialists were delivered with permission from the Society of Korean Medicine Pediatrics and the Society of Korean 
Medicine Neuropsychiatry. Registered mail was used for KMDs working at KM clinics for pediatric disease or psychiatric disease; correspondence was accompanied by a cover letter and freepost reply envelope.

The present study was approved by the institutional review board of Kyung Hee University Korean Medicine Hospital at Gangdong in Korea (KHNMCOH 2016-11008-002). All investigators adhered to the Helsinki Declaration. Respondents signed written informed consent forms to participate, explaining research background, purpose, methods, and personal information protection.

\section{Statistical analyses}

Using descriptive statistics, categorical data were presented by frequencies (percentages, \%) to describe the participants and their practices. Items that allowed multiple responses were evaluated using multiple response analysis. Results were analyzed with Microsoft Office Excel version 14.0 (Microsoft Corporation, Redmond, WA, USA).

\section{Results}

A total of $22.4 \%$ KMDs $(n=57 / 255)$ completed the questionnaire (16 face-to-face, 25 by registered mail, and 16 by e-mail). However, two respondents did not return the consent forms, and one was in the residency program and had never treated patients with ASD. Thus, 54 KMDs (21.2\%) matched the inclusion criteria.

In the current study, $61.1 \%$ of respondents were men, $48.1 \%$ were aged $30-39$ years, and $44.4 \%$ had $10-19$ years of experience as a KMD. A total $83.3 \%$ were specialists certified by the Korean Ministry of Health and Welfare. Among them, 60\% were certified by the Society of Korean Medicine Neuropsychiatry, and 40\% were licensed by the Society of Korean Medicine Pediatrics. For most KMDs (83.3\%), the proportion of patients with ASD among the total outpatients was less than $5 \%$. The main age group of patients treated by respondents was aged 6-10 years $(47.7 \%)$ (Table 1$)$.

KM syndrome differentiation was generally used $(88.9 \%)$ in the diagnostic process. The most frequently answered KM syndrome differentiation theory was 'Organ system, Qi, Blood, Yin, Yang, Fluid, and Humor diagnosis' (68.2\%), followed by 'Four-constitutional medicine diagnosis' (18.2\%), and 'Six meridian diagnosis' $(10.6 \%)$. Of the titles of syndrome differentiation on ASD, 'Heart deficiency with timidity (心虛膽怯)' (12.8\%) was most frequently used, followed by 'Liver qi depression (肝盬氣結)' (10\%), and 'Phlegm confounding the orifices of the heart (痰迷心竅)' (10\%). ASD was commonly diagnosed based on the fourth edition of the Diagnostic and Statistical Manual for Mental Disorders (DSM-IV; 26.7\%) and DSM-5 (23.8\%) (Table 2).
The most frequently prescribed KM treatment for ASD was herbal medicine (27.3\%), followed by body acupuncture (17.6\%), scalp acupuncture (10.7\%), and moxibustion (6.4\%). The main targets of KM treatment were to alleviate emotional problems (21.7\%), improve physical health (18.1\%), or improve cognitive ability (16.7\%). The most commonly used herbal medicines were Yukmijihwang-tang (12.4\%), Ondam-tang (11.2\%), and Gwibi-tang (7.7\%). Among the single herbs, Acori Graminei Rhizoma (9.5\%), Polygalae Radix (8.5\%), and Poria (7\%) were frequently used. KMDs mainly prescribed compound herbal decoction (55.7\%) among the various types of herbal medicine. Herbal medicine for ASD was commonly prescribed to be taken twice a day $(61.8 \%)$, with a treatment duration of 3-6 months (40.4\%), 13 months (21.1\%), or 6 months to 1 year (21.1\%). Regarding acupuncture treatment, GV20 (15.4\%), LI4 (11.7\%), and Ex-HN1 (Sasinchong, Sishencong) points (8.1\%) were the most widely used acupoints. The most commonly applied pharmacopuncture was Hominis Placenta (10.6\%) (Table 3). KMDs mainly performed body acupuncture twice (43.8\%) or once $(37.5 \%)$ a week. Scalp acupuncture was also conducted twice $(50 \%)$ or once $(34.6 \%)$ a week. The most predominant duration of body acupuncture treatment was 3-6 months (36.6\%) or more than 1 year $(31.7 \%)$. Scalp acupuncture was mainly applied for more than 1 year $(34.8 \%)$ or 3-6 months (26.1\%).

\section{Discussion}

ASD is a lifetime disease and causes a heavy burden to both families and society. The economic cost for children with ASD in the United States is between 11.560.9 billion USD, including healthcare and special education costs, and lost income [20, 21]. No single intervention has been confirmed as being effective in relieving the main symptoms of ASD, which is one reason why families of patients with ASD seek CAM [22, 23]. It was reported that $30-74 \%$ of children with ASD used CAM [24-26]. There are published reports on KM treatment for ASD, including herbal medicine and acupuncture, such as case reports [13, 14, 27] and reviews [28, 29]. However, the status of KM or CAM use on children with ASD has not yet been investigated in Korea. The present research was conducted to report the current state of KM treatment for children with ASD.

The rate of ASD among the outpatients that responded was less than $5 \%$, which might be related to the low prevalence of ASD [2,3]. In addition, KM may be used infrequently to treat ASD in Korea [30-32]. Patients with ASD treated by the respondents were predominantly aged $3-5$ or $6-10$ years, when multiple responses were allowed. This may have relevant to the 
Table 1 Sociodemographic characteristics of surveyed Korea medicine doctors

\begin{tabular}{|c|c|c|}
\hline \multicolumn{2}{|l|}{ Factors } & \multirow{2}{*}{$\frac{N(\%)}{0(0)}$} \\
\hline Age (years) & $20-29$ & \\
\hline & $30-39$ & $26(48.1)$ \\
\hline & $40-49$ & $23(42.6)$ \\
\hline & $50-59$ & $5(9.3)$ \\
\hline & $\geq 60$ & $0(0)$ \\
\hline \multirow[t]{2}{*}{ Sex } & Male & $33(61.1)$ \\
\hline & Female & $21(38.9)$ \\
\hline \multirow[t]{4}{*}{ Clinical experience (years) } & $\leq 4$ & $3(5.6)$ \\
\hline & $5-9$ & $16(29.6)$ \\
\hline & $10-19$ & $24(44.4)$ \\
\hline & $\geq 20$ & $11(20.4)$ \\
\hline \multirow{2}{*}{$\begin{array}{l}\text { Level of healthcare facility of currently } \\
\text { affiliated institution }\end{array}$} & Primary healthcare institution & $26(48.1)$ \\
\hline & Secondary healthcare institution & $28(51.9)$ \\
\hline \multirow[t]{4}{*}{ Place of work } & Capital & $21(38.9)$ \\
\hline & Metropolitan city & $10(18.5)$ \\
\hline & City & $23(42.6)$ \\
\hline & Country & $0(0)$ \\
\hline \multirow[t]{2}{*}{ Specialist training } & Yes & $45(83.3)$ \\
\hline & No & $9(16.7)$ \\
\hline \multirow[t]{2}{*}{ Medical specialty } & The Society of Korean Medicine Neuropsychiatry & $27(60)$ \\
\hline & The Society of Korean Medicine Pediatrics & $18(40)$ \\
\hline \multirow{5}{*}{$\begin{array}{l}\text { Rate of autism spectrum disorder patients among } \\
\text { total outpatients (\%) }\end{array}$} & $<5$ & $45(83.3)$ \\
\hline & $5-9$ & $5(9.3)$ \\
\hline & $10-19$ & $2(3.7)$ \\
\hline & $20-49$ & $1(1.9)$ \\
\hline & $\geq 50$ & $1(1.9)$ \\
\hline \multirow{5}{*}{$\begin{array}{l}\text { Age of patients with ASD treated by respondents (years) } \\
\text { (multiple responses allowed) }\end{array}$} & $<3$ & $7(8.1)$ \\
\hline & $3-5$ & $27(31.4)$ \\
\hline & $6-10$ & $41(47.7)$ \\
\hline & $11-20$ & $9(10.5)$ \\
\hline & $\geq 21$ & $2(2.3)$ \\
\hline
\end{tabular}

fact that most children are not diagnosed with ASD until after 4 years of age [2].

Most KMDs answered that they were using KM syndrome differentiation. Syndrome differentiation is a type of diagnostic method that decides the syndrome based on a group of coexistent symptoms and signs [33]. After syndrome differentiation is decided, the KMDs select the treatment method, including herbal medicine and acupuncture. Syndrome differentiation enables KM treatment to fit individual physical features, and signs and symptoms. When KMDs examine a patient and determine a diagnosis, they significantly consider diseases identification (辨病) as well as syndrome differentiation [34]. In the current study, most KMDs replied that they diagnosed ASD based on DSM-IV and DSM-5. Some KMDs did not use diagnostic tools but decided to conduct the diagnosis based on symptoms. For accurate diagnosis and assessment of treatment, it is recommended that standardized diagnostic tools and appropriate evaluation indexes should be used.

The respondents preferred to use herbal medicine, mostly choosing compound herbal decoction when prescribing herbal medicine. When prescribing compound herbal decoction, KMDs can mix several kinds of herbs in varying amounts, and it can be more effective and helpful for patients with different symptoms and constitutional characteristics. The most frequently prescribed herbal medicine was Yukmijihwang-tang. The memory 
Table 2 Korean Medicine syndrome differentiations and diagnostic tools used for autism spectrum disorder

\begin{tabular}{|c|c|c|}
\hline \multicolumn{2}{|l|}{ Factors } & \multirow{2}{*}{$\frac{N(\%)}{48(88.9)}$} \\
\hline Do you use Korean Medicine syndrome differentiation? & Yes & \\
\hline & No & $6(11.1)$ \\
\hline \multirow[t]{4}{*}{$\begin{array}{l}\text { Korean Medicine syndrome differentiation theories } \\
\text { (multiple responses allowed) }\end{array}$} & $\begin{array}{l}\text { Organ system, Qi, Blood, Yin, Yang, Fluid and Humor diagnosis } \\
\text { (臟腑氣血陰陽津液辨證) based on KM textbooks }\end{array}$ & $45(68.2)$ \\
\hline & $\begin{array}{l}\text { Four-constitutional medicine diagnosis (四象醫學辨證)' } \\
\text { based on 'Dongeuisoosebowon (東醫壽世保元) }\end{array}$ & $12(18.2)$ \\
\hline & $\begin{array}{l}\text { Six meridian diagnosis (六經辨證) based on } \\
\text { 'Shang Han Lun (傷寒論)' }\end{array}$ & $7(10.6)$ \\
\hline & Meridian system diagnosis (經絡辨證) & $2(3)$ \\
\hline \multirow{10}{*}{$\begin{array}{l}\text { Title of frequently used syndrome differentiation } \\
\text { (multiple responses allowed) }\end{array}$} & Heart deficiency with timidity (心虛膽怯) & $23(12.8)$ \\
\hline & Liver qi depression (肝㯺氣結) & $18(10)$ \\
\hline & Phlegm confounding the orifices of the heart (痰迷心竅) & $18(10)$ \\
\hline & Dual deficiency of heart and spleen (心脾兩虛) & $18(10)$ \\
\hline & Qi deficiency (氣虛) & $14(7.8)$ \\
\hline & Kidney essence deficiency (腎精虧虛) & $14(7.8)$ \\
\hline & Dual deficiency of qi and blood (氣血兩虛) & $12(6.7)$ \\
\hline & Liver-kidney yin deficiency (肝腎陰虛) & $12(6.7)$ \\
\hline & Kidney qi insufficiency (腎氣不足) & $12(6.7)$ \\
\hline & Blood deficiency (血虛) & $11(6.1)$ \\
\hline \multirow[t]{5}{*}{$\begin{array}{l}\text { Diagnostic tool } \\
\text { (multiple responses allowed) }\end{array}$} & $\begin{array}{l}\text { the Diagnostic and Statistical Manual for Mental Disorders, } \\
\text { fourth edition (DSM-IV) }\end{array}$ & $28(26.7)$ \\
\hline & $\begin{array}{l}\text { the Diagnostic and Statistical Manual for Mental Disorders, } \\
\text { fifth edition (DSM-5) }\end{array}$ & $25(23.8)$ \\
\hline & Not using diagnostic tools but deciding by symptoms & $16(15.2)$ \\
\hline & $\begin{array}{l}\text { Not using diagnostic tools but referral to other hospital } \\
\text { or specialist }\end{array}$ & $15(14.3)$ \\
\hline & Childhood Autism Rating Scale (CARS) & $10(9.52)$ \\
\hline
\end{tabular}

enhancing and learning function of Yukmijihwang-tang has been demonstrated in laboratory studies [35, 36], with published case studies about herbal medicine containing Yukmijihwang-tang for ASD treatment [13, 14]. Clinical studies on the use of Yukmijihwang-tang in pediatric patients, however need to conducted using rigorous methodology.

Patients with ASD have diverse symptoms, with varying degrees of impairment in social and communication function. KMDs mostly targeted the reduction of emotional problems such as anxiety, irritation, and moodiness. Improving physical condition, associated with food intake, digestion, defecation, and sleep, was also an important therapeutic goal. Gastrointestinal symptoms accounted for a high percentage of the CAM used for children with ASD in the United States [37]. To accurately evaluate the overall therapeutic effect, several assessment indicators for various symptoms will be needed.

Our study has some limitations that need to be noted. One of the most general methods of surveying KMDs is to send e-mails containing a web-based questionnaire to all KMDs through the Associations of Korean Medicine. Nevertheless, this approach is known to have low response rates (3.9-11.1\%) [38, 39]. Patients chiefly visit a KM clinic to be treated for musculoskeletal disorders, and the frequency of KM treatment of mental disorders is low in Korea [11]. Thus, we postulated that there was a high probability that general KMDs would not respond to the survey. Therefore, we did not target all KMDs, but sent e-mails to all KM psychiatrists and pediatricians through the Society of Korean Medicine Neuropsychiatry and Society of Korean Medicine Pediatrics, and KMDs who were found by internet searching. Thus, this process may have had a selection bias for KMDs who use e-mails or run a website. Therefore, there may have been some KMDs who treat ASD that were excluded from the analysis. In addition, our study does not show the effect of each of interventions on core symptoms of ASD. Future studies would entail researching the efficacy and safety of specific KM treatments for patients with ASD. Further, the standpoint of families on using KM treatment for ASD has not been examined. Thus, a study on caregiver perceptions about the efficacy, 
Table 3 Frequently prescribed Korean medicine treatments for autism spectrum disorder

\begin{tabular}{|c|c|c|}
\hline Factor & & $\mathrm{N}(\%)$ \\
\hline \multirow{8}{*}{$\begin{array}{l}\text { Treatment } \\
\text { (multiple responses allowed) }\end{array}$} & Herbal medicine & $51(27.3)$ \\
\hline & Body acupuncture & $33(17.6)$ \\
\hline & Scalp acupuncture & $20(10.7)$ \\
\hline & Moxibustion & $12(6.4)$ \\
\hline & Korean medical psychotherapy & $11(5.9)$ \\
\hline & Chuna manual therapy & $10(5.4)$ \\
\hline & Neurofeedback & $7(3.7)$ \\
\hline & Psychotherapy & $7(3.7)$ \\
\hline \multirow{6}{*}{$\begin{array}{l}\text { Treatment target } \\
\text { (multiple responses allowed) }\end{array}$} & Emotional problems & $48(21.7)$ \\
\hline & Physical condition & $40(18.1)$ \\
\hline & Cognitive ability & $37(16.7)$ \\
\hline & Behavior problems & $34(15.4)$ \\
\hline & Social interaction & $33(14.9)$ \\
\hline & Communication ability & $27(12.2)$ \\
\hline \multirow{7}{*}{$\begin{array}{l}\text { Herbal medicine } \\
\text { (multiple responses allowed) }\end{array}$} & Yukmijihwang-tang (六味地黄湯) & $21(12.4)$ \\
\hline & Ondam-tang (溫膽湯) & $19(11.2)$ \\
\hline & Gwibi-tang (歸脾湯) & $13(7.7)$ \\
\hline & Eokgan-san (抑肝散) & $11(6.5)$ \\
\hline & Sihogayonggolmoryeo-tang (柴胡加龍骨牡蠣湯) & $10(5.9)$ \\
\hline & Bojungikgi-tang (補中益氣湯) & $8(4.7)$ \\
\hline & Soyo-san (逍遙散) & $6(3.6)$ \\
\hline \multirow{9}{*}{$\begin{array}{l}\text { Single herb } \\
\text { (multiple responses allowed) }\end{array}$} & Acori Graminei Rhizoma (石菖蒲) & $19(9.5)$ \\
\hline & Polygalae Radix (遠志) & $17(8.5)$ \\
\hline & Poria (白获笭) & $14(7)$ \\
\hline & Bupleuri Radix (柴胡) & $10(5)$ \\
\hline & Rehmanniae Radix Preparat (熟地黄) & $9(4.5)$ \\
\hline & Cyperi Rhizoma (香附子) & $8(4)$ \\
\hline & Fossilia Ossis Mastodi (龍骨) & $7(3.5)$ \\
\hline & Ostreae Concha (牡蠣) & $7(3.5)$ \\
\hline & Angelicae Gigantis Radix (當歸) & $7(3.5)$ \\
\hline \multirow{7}{*}{$\begin{array}{l}\text { Acupoint used for acupuncture } \\
\text { (multiple responses allowed) }\end{array}$} & GV20 (百會) & $42(15.4)$ \\
\hline & LI4 (合谷) & $32(11.7)$ \\
\hline & Ex-HN1 (四神聰, Sasinchong, Sishencong) points & $22(8.1)$ \\
\hline & HT7 (神門) & $21(7.7)$ \\
\hline & LR3 (太衝) & $20(7.3)$ \\
\hline & ST36 (足三里) & $18(6.6)$ \\
\hline & PC6 (內關) & $16(5.9)$ \\
\hline \multirow{4}{*}{$\begin{array}{l}\text { Pharmacopuncture } \\
\text { (multiple responses allowed) }\end{array}$} & Not using pharmacopuncture & $28(59.6)$ \\
\hline & Hominis Placenta (紫河車) & $5(10.6)$ \\
\hline & Ginseng (山蔘) & $4(8.5)$ \\
\hline & Hwangryeonhaedok (黄連解毒) & $4(8.5)$ \\
\hline
\end{tabular}


expectation and rationale for using $\mathrm{KM}$ treatment is warranted.

The questionnaire employed also has several limitations. There were a lot of items to answer and the length of the questionnaire was long. This may account for missing data. When asked about the type of pharmacopuncture used, 18 KMDs stated the type of pharmacopuncture used, 28 people replied that they did not use pharmacopuncture, and 8 KMDs did not answer this question. We excluded missing data and performed a multiple response analysis. Second, the validity of the questionnaire was partially verified. We searched former survey studies [7, 40, 41] on KM treatment, but did not find validated questionnaires. Accordingly, we derived and modified a considerable number of items from several studies. A panel of experts reviewed the questionnaire, but statisticians or methodologists were not included in the panel. Third, we allowed respondents to provide multiple responses to most items because we intended for the survey to reflect the reality that KMDs judge and change the treatment method according to the circumstances. Another reason for allowing plural responses was that KM treatment was not highly standardized for ASD.

This is the first survey report on clinical practice pattern of KM treatment of ASD. The draft of CPG for KM treatment of ASD was completed in June 2017, and preliminary certification by the Guideline Center for Korean Medicine was obtained in September 2017; it is yet to be published. When establishing a CPG for ASD, the research team reflected on the findings of the current study and made recommendations for each diagnosis and treatment method. Based on the survey results, detailed information was also included that can be used when KMDs treat patients with ASD. In the course of the preparation of the CPG, the research team collected and analyzed practice guidelines and literatures on ASD, and systematically reviewed the efficacy and safety of herbal medicine [42] and acupuncture [43]. In addition, a randomized controlled trial using an herbal medicine, which the present study identified as being widely used, has been scheduled for 2018. The future CPG will be used as an evidence-based guideline in KM institutions, helping KMDs make decisions about ASD and improving the quality and reliability of medical treatment.

\section{Conclusions}

The present study demonstrated the current status of KMDs' clinical practice patterns for ASD. KMDs commonly used KM syndrome differentiations when diagnosing patients with ASD patients; the 'Organ system, Qi, Blood, Yin, Yang, Fluid and Humor diagnosis' was most frequently used among KM syndrome differentiation theories. In addition, herbal medicine and body acupuncture are the most employed KM interventions. Among herbal medicines, Yukmijihwang-tang was the most frequently prescribed. The data of this survey will serve as the basis for CPGs and future clinical trials on ASD. Standardized and reliable CPGs will enable KMDs and patients make rational clinical decisions.

\section{Additional file}

Additional file 1: Survey of Korea Medicine doctors' clinical patterns for autism spectrum disorder. The final questionnaire answered by KMDs (DOCX $39 \mathrm{~kb})$

\section{Abbreviations}

ASD: Autism Spectrum Disorder; CAM: Complementary and alternative medicine; CPGs: Clinical practice guidelines; DSM-IV: The fourth edition of the Diagnostic and Statistical Manual for Mental Disorders; KM: Korean medicine; KMD: Korean Medicine doctor; MD: Medical doctor

\section{Acknowledgements}

This study was supported by theTraditional Korean Medicine R\&D program funded by the Ministry of Health \& Welfare through the Korea Health Industry Development Institute (KHIDI) (HB16C0075).

Availability of data and materials

All the data are presented within the manuscript.

\section{Authors' contributions}

$J$, SHL, BL, and GTC constructed and revised the questionnaire. $J$ analyzed the data and wrote the manuscript. SHL, BL, and GTC acquired and analyzed the data, and reviewed the manuscript. IJY contributed to the work-related statistics, reviewed and revised the manuscript. All authors read and approved the final manuscript.

\section{Ethics approval and consent to participate}

Ethical approval was obtained from the institutional review board of Kyung Hee University Korean Medicine Hospital at Gangdong in Korea (KHNMCOH 2016-11-008-002). All investigators adhered to the Helsinki Declaration. Respondents signed written informed consent forms to participate, explaining research background, purpose, methods, and personal information protection.

\section{Consent for publication}

Not applicable. Our manuscript does not contain any data from individual person. Our manuscript is not a report of medical cases that include individual details, pictures or videos. In our survey research, individual information is not required, and statistical analysis is performed with raw survey data.

\section{Competing interests}

The authors declare that they have no competing interests.

\section{Publisher's Note}

Springer Nature remains neutral with regard to jurisdictional claims in published maps and institutional affiliations.

\section{Author details}

${ }^{1}$ Department of Clinical Korean Medicine, Graduate School, Kyung Hee University, Seoul 02453, Republic of Korea. ${ }^{2}$ Department of Pediatrics of Korean Medicine, Kyung Hee University Hospital at Gangdong, \#892 Dongnam-ro, Gangdong-gu, Seoul 05278, Republic of Korea. ${ }^{3}$ Department of Pediatrics of Korean Medicine, Kyung Hee University Korean Medicine Hospital, Kyung Hee University Medical Center, Seoul 02447, Republic of Korea. ${ }^{4}$ Department of Physiology, College of Korean Medicine Dongguk University, Gyeongju 780-714, Republic of Korea. 


\section{Received: 11 September 2017 Accepted: 8 March 2018} Published online: 13 March 2018

\section{References}

1. American Psychiatric Association. Autism spectrum disorder. In: Diagnostic and statistical manual of mental disorders. 5th ed. Seoul: Hakjisa; 2013. p. 50-60.

2. Christensen DL, Baio J, Braun KVN, Bilder D, Charles J, Constantino JN, Daniels J, Durkin MS, Fitzgerald RT, Margaret KS, Lee LC, Pettygrove S, Robinson C, Schulz E, Wells C, Wingate MS, Zahorodny W, Marshalyn YA. Prevalence and characteristics of autism spectrum disorder among children aged 8 years-autism and developmental disabilities monitoring network, 11 sites, United States, 2012. MMWR Surveill Summ. 2016:65(3):1-23.

3. Kim YS, Leventhal BL, Koh Y, Fombonne E, Laska E, Lim E, Cheon K, Kim S, Kim YK, Lee H, Song D, Grinker RR. Prevalence of autism spectrum disorders in a total population sample. Am J Psychiatry. 2011;168:904-12.

4. Myers SM, Johnson CP. Management of children with autism spectrum disorders. Pediatrics. 2007:120:1162-82.

5. Park WS, Mollahaliloglu S, Linnik V, Chae H. Scientific evidence for Korean medicine and its integrative medical research. Evid Based Complement Alternat Med. 2015; https://doi.org/10.1155/2015/967087.

6. Park HL, Lee HS, Shin BC, Liu JP, Shang QH, Yamashita H, Lim BM. Traditional medicine in China, Korea, and Japan: a brief introduction and comparison. Evid Based Complement Alternat Med. 2012; https://doi.org/10. 1155/2012/429103.

7. Shin Y, Shin JS, Lee J, Lee YJ, Kim M, Ahn Y, Park KB, Shin BC, Lee MS, Kim $\mathrm{JH}, \mathrm{Cho} \mathrm{JH}, \mathrm{Ha} \mathrm{IHA}$. Survey among Korea medicine doctors (KMDs) in Korea on patterns of integrative Korean medicine practice for lumbar intervertebral disc displacement: preliminary research for clinical practice guidelines. BMC Complement Altern Med. 2015;15:432.

8. Oh $\mathrm{H}$, Yoon SJ, Park M, An S. Disease-specific differences in the use of traditional Korean medicine in Korea. BMC Complement Alternat Med. 2015;15:41.

9. Open Data Portal. https://www.data.go.kr/dataset/15003224/fileData.do. Accessed 15 Jan 2018

10. 2016 survey on the Korean medicine industry. Korea institute of oriental medicine, 2016. https://www.kiom.re.kr/brdartcl/boardarticleView.do?menu nix=WUNNW2Aq\&brd_id=BDIDX_o9YEVvNb40b134N1Rt17aq\&cont_idx=6. Accessed 16 Jan 2018.

11. Cheong M, Park J, Seo K, Huang J, Kim E, Kim J, Han K, Kim S, Kim Y. National survey on the use of Korean medicine and Korean herbal medicine. Korea Health Ind Dev Inst. 2014;11-1352000-000547-12:1-778.

12. Institute of Medicine (US) Committee to Advise the Public Health Service on Clinical Practice Guidelines. Clinical practice guidelines: directions for a new program. Washington (DC): National Academies Press (US); 1990.

13. Lee JN, Kim DG, Lee JY. Report on seven cases on patients with autism spectrum disorder treated by Kwakhyangjungkisanhapyukmijihwangtanggamibang. J Pediatr Korean Med. 2015;29(1):50-9.

14. Jang JH, Lim YW, Lee SY. Two cases report about atypical autistic children. J Pediatr Korean Med. 2005;19(2):85-97.

15. Google. http://www.google.co.kr. Accessed 20 Sept 2016

16. Naver. http://www.naver.com. Accessed 20 Sept 2016.

17. Daum. http://www.daum.net. Accessed 20 Sept 2016.

18. Korean Society of Oriental Neuropsychiatry. Oriental neuropsychiatry. Seoul: Jip Moon Dang; 2010.

19. Kim KB, Kim DG, Kim YH, Kim JH, Min SY, Park EJ, Baek JH, Sung HK, Yu SA Lee SY, Lee JY, Lee HJ, Chang GT, Jeong MJ, Chai JW, Cheon JH, Han YJ, Han JK. Korean pediatrics \& adolescent medicine. Ui Sung Dang: Seoul; 2015

20. Lavelle TA, Weinstein MC, Newhouse JP, Munir K, Kuhlthau KA, Prosser LA. Economic burden of childhood autism spectrum disorders. Pediatrics. 2014; 133:e520-9.

21. Buescher AVS, Cidav Z, Knapp M, Mandell DS. Costs of autism spectrum disorders in the United Kingdom and the United States. JAMA Pediatr. 2014; 168(8):721-8.

22. Hyman SL, Levy SE. Autistic spectrum disorders: when traditional medicine is not enough. Contemp Pediatr. 2000;17(10):101.

23. Sandler AD, Brazdziunas D, Cooley WC, Gonzalez de Pijem L, Hirsch D, Kastner TA, Kummer ME, Quint RD, Ruppert ES. Counseling families who choose complementary and alternative medicine for their child with chronic illness or disability. Pediatrics. 2001;107(3):598-601.
24. Levy SE, Mandell DS, Merhar S, Ittenbach RF, Pinto-Martin JA. Use of complementary and alternative medicine among children recently diagnosed with autistic spectrum disorder. J Dev Behav Pediatr. 2003;24(6):418-22.

25. Levy SE, Hyman SL. Alternative/complementary approaches to treatment of children with autistic spectrum disorders. Infants Young Child. 2002;14(3): 33-42.

26. Nickel RE. Controversial therapies for young children with developmental disabilities. Infants Young Child. 1996;8(4):29-40.

27. Hong SJ, Choi KH, Song IS. A clinical report of scalp acupuncture effects on autistic children. J Pediatr Korean Med. 2009;23(3):133-42.

28. Lee JH, Han JK, Lee HL, Kim YH. Review of clinical studies for oriental treatment of autism in traditional chinese medicine. J Pediatr Korean Med. 2014;28(4):85-95.

29. Lee JN, Lee SH, Lee JY. Recent clinical research on effect of acupuncture for autism spectrum disorder. J Pediatr Korean Med. 2015;29(4):119-26.

30. Lee EJ, Lee BR, Lee JH, Chang GT. A study on the recent trend of chief complaint of Korean pediatric and adolescent outpatients. J Pediatr Korean Med. 2016;30(1):45-58

31. Choi MH, Kim DG, Lee JY. A study of the chief complaint of pediatric outpatients in the Kyung Hee oriental medicine hospital. J Pediatr Korean Med. 2010;24(3):121-37.

32. Yoon KH, Koh DJ, Kim DG. Research on the actual conditions of the outpatients who visited the pediatrics department in $\circ \circ$ university oriental medicine hospital between 2005 and 2006. J Pediatr Korean Med. 2007; 21(3):125-43.

33. Yun $Y$, Jung $W$, Kim H, Jang BH, Kim MH, Noh J, Ko SG, Choi I. Exploring syndrome differentiation using non-negative matrix factorization and cluster analysis in patients with atopic dermatitis. Comput Biol Med. 2017;87:70-6.

34. Choi SH. Korean medicine. Kyobo Book Centre. 2016; http://www.bigbook. or.kr/bbs/bbs/board.php?bo_table=bo16\&sca=\&sfl=wr_subject\&stx= $\% C 7 \% D 1 \% C 0 \% C 7 \% C 7 \% D 0 \& x=0 \& y=0$. Accessed 22 July 2017

35. Rho S, Kang M, Choi B, Sim D, Lee J, Lee E, Cho C, Oh J, Park S, Ko S, Shin $\mathrm{M}$, Hong $\mathrm{M}$, Bae $\mathrm{H}$. Effects of Yukmijihwang-tang derivatives (YMJd), a memory enhancing herbal extract, on the gene-expression profile in the rat hippocampus. Biol Pharm Bull. 2005:28(1):87-93.

36. Huang Y, Zhang H, Yang S, Qiao H, Zhou W, Zhang Y. Liuwei Dihuang decoction facilitates the induction of long-term potentiation (LTP) in senescence accelerated mouse/prone 8 (SAMP8) hippocampal slices by inhibiting voltage-dependent calcium channels (VDCCs) and promoting $\mathrm{N}$-methyl-d-aspartate receptor (NMDA) receptors. J Ethnopharmacol. 2012;140:384-90.

37. Perrin JM, Coury DL, Hyman SL, Cole L, Reynolds AM, Clemons T. Complementary and alternative medicine use in a large pediatric autism sample. Pediatrics. 2012;130(Suppl):77.

38. Park B, Yun KJ, Jung J, You S, Lee JA, Choi J, Kang BK, Alraek T, Birch S, Lee MS. Conceptualization and utilization of blood stasis syndrome among doctors of Korean medicine: results of a web-based survey. Am J Transl Res. 2014;6(6):857-68.

39. Ko M, Lee J, Yun K, You S, Lee M. Perception of pattern identification in traditional medicine: a survey of Korean medical practitioners. J Tradit Chin Med. 2014;34(3):369-72.

40. Yang C, Lee E, Hwang E, Kwon O, Lee J. Management of sport injuries with Korean medicine: a survey of Korean national volleyball team. Evid Based Complement Alternat Med. 2016; https://doi.org/10.1155/2016/8639492.

41. Son $\mathrm{CH}$, Yun YJ, Yu SA. The experts survey and in-depth interview on the Korean medicine treatments for the cerebral palsy children. J Pediatr Korean Med. 2016:30(4):66-76.

42. Bang M, Lee SH, Cho SH, Yu SA, Kim K, Lu HY, Chang GT, Min SY. Herbal medicine treatment for children with autism spectrum disorder: a systematic review. Evid Based Complement Alternat Med. 2017; https://doi. org/10.1155/2017/8614680.

43. Lee B, Lee J, Cheon JH, Sung HK, Cho SH, Chang GT. Efficacy and safety of acupuncture for the treatment of children with autism spectrum disorder: a systematic review and meta-analysis. Evid Based Complement Alternat Med. 2018; https://doi.org/10.1155/2018/1057539. 